\title{
Running-in of a Double Network Gel for Low Friction in Water
}

\author{
Koki Kanda* and Koshi Adachi \\ Department of Mechanical Systems Engineering, Tohoku University, \\ 6-6-01 Aramaki-Aza-Aoba, Aoba-ku, Sendai, Miyagi 980-8579, Japan \\ ${ }^{*}$ Corresponding author: Koki Kanda (koki.kanda.a5@tohoku.ac.jp)
}

Manuscript received 25 April 2021; accepted 05 August 2021; published 15 September 2021

\begin{abstract}
This study aims to clarify the potential of double network (DN) gel to realize low friction coefficient under water lubrication environment. Friction tests were carried out by using DN gel film and silicon carbide (SiC) disk in water. DN gel shows runningin behavior consists of lowering friction coefficient twice, then friction coefficient shows less than 0.001 under limited experimental conditions. By introducing in-situ test apparatus, self-formation of damaged region is confirmed and external water penetrates into selfformed damaged region. As a result, damaged region becomes water-rich situation more and more and formation of lubricious water layer is enhanced gradually. Thus running-in behavior occurs by friction system using DN gel.
\end{abstract}

\section{Keywords}

double network gel, damaged zone, low friction, self-formation

\section{Introduction}

Running-is the phenomena that indicates saturation of friction and/or wear using solid materials and determined by several surface factors, such as surface property, environmental property and kinetic property of the tribological system [1, 2]. Thus running-in phenomena is focused as new revolution to realize excellent working property of machine elements. Especially low friction and low wear $[3,4]$ are main topic of running-in study since both of them are required for ideal machine elements. Mechanism of running-is widely explained by realized hydrodynamic lubrication due to wear and smoothing surfaces [5], chemical reaction [6] and formation of boundary films which shows low friction [7] due to raised pressure or temperature caused at actual contact point on surfaces in friction situation

In other words, change of surface state enables running-in, therefore recent trend is shifting to design initial surface more reactive to enhance running-in and to improve performance after running in is finished [8].

As next-generation machine elements, gel materials [9], consist of a polymer and a fluid, such as oil [10] or water [11], and a polymer network is formed by the physical or chemical cross-linking of the chemical chains, are expected to relieve stress due to their softness and elasticity. In addition, owing to their higher fluid content, gel materials are believed to attract similar fluids around them or to extrude fluids, which in turn can form a fluid film, from the inside to the outside of a gel material.
Previously it was reported that structure at near top surface affects effect on friction property of friction system using gel material, that is, sparse gel easily forms water interlayer between gel and sliding counterpart and results in low friction due to exuded water while cross-linked and rigid top surface hardly form it [12]. Also, rehydration enhances formation of water interlayer and decreases friction coefficient of friction system using hydrogels. [13]. These studies clearly indicate formation of water interlayer is crucial to realize low friction. Also, generation of low friction can be explained by alignment of hydrogel polymer chain along sliding direction [14]

Nowadays, double network (DN) gels, which consist of several different polymer networks, are preferred due to their high mechanical strength [15]. Several tribological studies using DN gels have been done. First, the importance of an external water lubricant, different from the water included inside the DN gel, to achieve low friction is demonstrated by resonance shear measurement (RSM) system [16]. Also previous study [17] revealed low friction can be realized by water layer between DN gel and sliding counterpart.

We focused DN gel material due to its weaker fracture toughness rather than that of hard metal or ceramic materials and tougher bulk strength compared to conventional soft matter such as PVA hydrogel. These factors seem to be ideal to cause reaction on top surface as well as prevent severe wear or catastrophic fracture as sliding materials. Thus this study aims to clarify the potential of DN gel to realize low friction by running-in behavior with changing top surface. 


\section{Experimental setup}

\subsection{Specimen}

A DN gel film was synthesized according to a previous publication [16]. Acrylamido-2-Methyl-1-PropaneSulfonic acid (AMPS, Wako Pure Chemical Industries, Ltd.) and N,NDiMethylAcrylAmide (DMAAm, Wako Pure Chemical Industries, Ltd.) were used as monomers in the first and second networks, respectively. N,N'-MethyleneBisAcrylAmide (MBAA, Wako Pure Chemical Industries, Ltd.) and $\alpha$-ketoglutaric acid (Wako Pure Chemical Industries, Ltd.) were used as crosslinkers and reaction initiators in each synthesis process. The detailed compositions of the first and second networks are shown in Table 1. The first network solution was stirred and poured into the mold consists of a glass plate and a silicon soft sheet which determined the thickness of the fabricated DN gel. Then, UV irradiation was used by a black light tube for $18 \mathrm{~h}$ to polymerize the first network. The second step of this process was that the polymerized first network gel film was immersed in a stirred second network solution for $48 \mathrm{~h}$ and then UV irradiation was used again for $18 \mathrm{~h}$ to polymerize the second network with the first network, subsequently fabricating a double network gel film. Finally, the double network gel film was cut into specimen shapes by a laser cutter (HAJIME, OhLaser Co., LTD). The diameter and thickness of the DN gel film

Table 1 List of chemicals and mol ratios in synthesis of (a) first network and (b) second network of DN gel

(a) first network

\begin{tabular}{|l|l|l|}
\hline Role & Name & Mol ratio \\
\hline Monomer & AMPS & 1 \\
\hline Cross-linker & MBAA & 0.04 \\
\hline Initiator & $\alpha$-ketoglutaric acid & 0.001 \\
\hline Solvent & Water & 43 \\
\hline
\end{tabular}

(b) second network

\begin{tabular}{|l|l|l|}
\hline Role & Name & Mol ratio \\
\hline Monomer & DMAAm & 1 \\
\hline Cross-linker & MBAA & 0.0002 \\
\hline Initiator & $\alpha$-ketoglutaric acid & 0.001 \\
\hline Solvent & Water & 22 \\
\hline
\end{tabular}

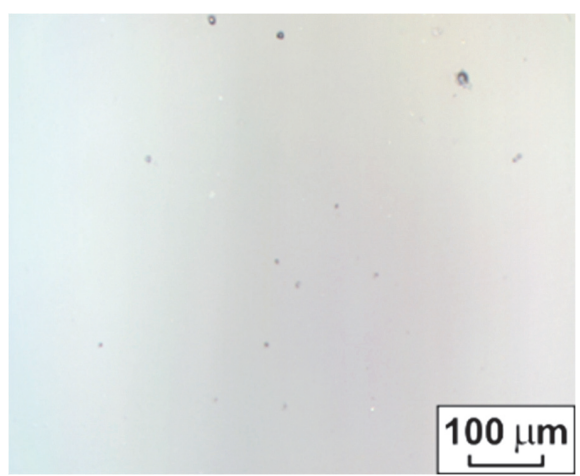

(a) DN gel were $10 \mathrm{~mm}$ and $2 \mathrm{~mm}$, respectively. All synthesis processes were carried out at $30^{\circ} \mathrm{C}$ in a thermostat chamber.

We also employed PVA gel film made based on previous publication [18] as representative materials of conventional hydrogels in order to highlight potential of DN gel. The initial images of the DN gel and the PVA gel surfaces were observed by a optical microscope (H1200, Lasertec Corporation), and are compared in Fig. 1. The DN gel surface appears flat although it contains some dispersed small concave spots initially, which have a diameter smaller than $10 \mu \mathrm{m}$, as observed. On the other hand, the initial surface of the PVA gel appears rough and consists of dense random concave spots and lines. Material parameter, such as young's modulus and water content of DN gel reported as 0.3 MPa [19] and 90 mass\% [15]. On the other hand, these of PVA hydrogel are $291 \mathrm{kPa}$ [20] and 15\%.

As a sliding counterpart for the DN gel film and PVA gel film, a disk made of silicon carbide $(\mathrm{SiC})$ was used. The diameter and thickness of the $\mathrm{SiC}$ disk were $30 \mathrm{~mm}$ and $4 \mathrm{~mm}$, respectively. The arithmetic surface roughness of the SiC disk was measured by surface probe microscopy (E-Sweep, Hitachi High-Technologies Co.) and found to be less than $10 \mathrm{~nm}$. The water contact angle on the SiC disk was measured by contact angle meter (DM-501, Kyowa Interface Science Co., Ltd.) and found to be $65^{\circ}$ approximately.

\subsection{Test apparatus}

The ball/disk friction apparatus was used to measure the friction force generated between the DN gel film and the $\mathrm{SiC}$ disk. The schematic image of the ball/disk friction apparatus is shown in Fig. 2. The SiC disk was fixed on a disk holder and rotated by a servo-controlled motor while the DN gel film was fixed on a $\mathrm{SiC}$ ball. The diameter of the SiC ball was

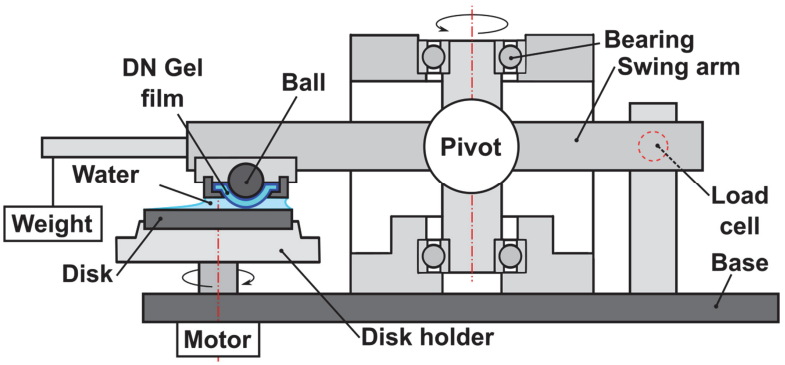

Fig. 2 Schematic image of the ball/disk friction apparatus

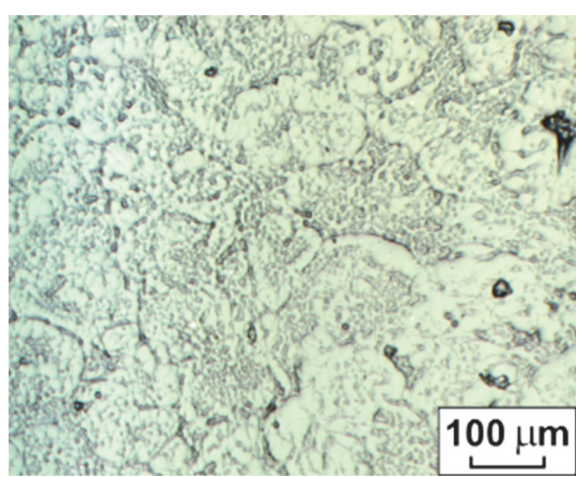

(b) PVA gel

Fig. 1 Initial surface images of (a) the DN gel and (b) the PVA gel 
$8 \mathrm{~mm}$. Contact load was applied by a mounted weight which generated a friction force that was measured by a load cell (LMA-A-5N, Kyowa Electronic Instruments Co., Ltd.) through a pivot and swing arm. Resolution of mounted load cell is $0.0025 \mathrm{~N}$, then resolution of friction will be 0.00025 when 10 $\mathrm{N}$ is applied. By considering friction loss around pivot system in the test apparatus, practical resolution of friction coefficient will be 0.001 approximately. In this study, $1 \mathrm{~N}, 5 \mathrm{~N}$, and $10 \mathrm{~N}$ forces were selected for the contact load. By observing contact area of DN gel through transparent glass, contact pressure for each loading cases are calculated as $0.10 \mathrm{MPa}, 0.19 \mathrm{MPa}$ and 0.36 $\mathrm{MPa}$, respectively. Range of sliding speed was varied from 1 $\mathrm{mm} / \mathrm{sec}$ to $500 \mathrm{~mm} / \mathrm{sec}$. Sliding distance of all experiments were set as $30 \mathrm{~m}$.

Adequate amounts of purified water were dropped around the contact region so that the $\mathrm{t}$ region between the $\mathrm{SiC}$ disk and the DN gel film was immersed in purified water through the whole duration of the experiment. The friction properties of the PVA gel were also investigated and compared with the results of the DN gel. All experimental procedures were carried out at room temperature.

The sliding test was conducted ones for each sliding test condition.

\section{Experimental results}

\subsection{Friction property}

The typical friction properties of the DN gel that slid against the SiC disk in purified water are shown in Fig. 3. The friction properties are classified into four modes based on their frictional behavior regardless of wear morphology, as follows:

Mode I: The friction coefficient is stable through of the experiment.

Mode II: The friction decreases and has stable value.

Mode III: The friction coefficient decreases twice.

Mode IV: The friction coefficient cannot be measured since the value is lower than the detection limit of the friction apparatus that was used.

The typical friction properties when the PVA gels were paired with the SiC disk are shown in Fig. 4. The sliding speed and load are set to similar values as those of Fig. 3. When the PVA gel films were examined, mode I, II and III appeared similar as well as DN gel. Modes IV did not take place around the investigated experimental conditions in this study.

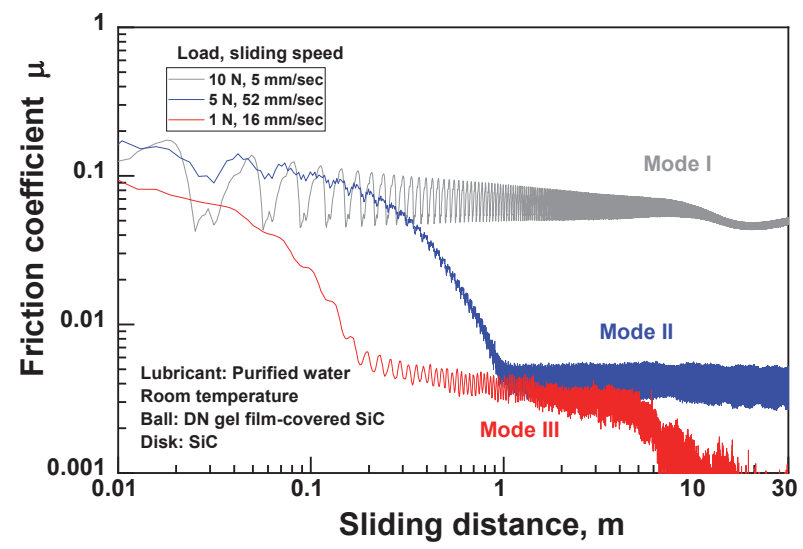

Fig. 3 Typical friction properties of $\mathrm{DN}$ gel and $\mathrm{SiC}$ in water

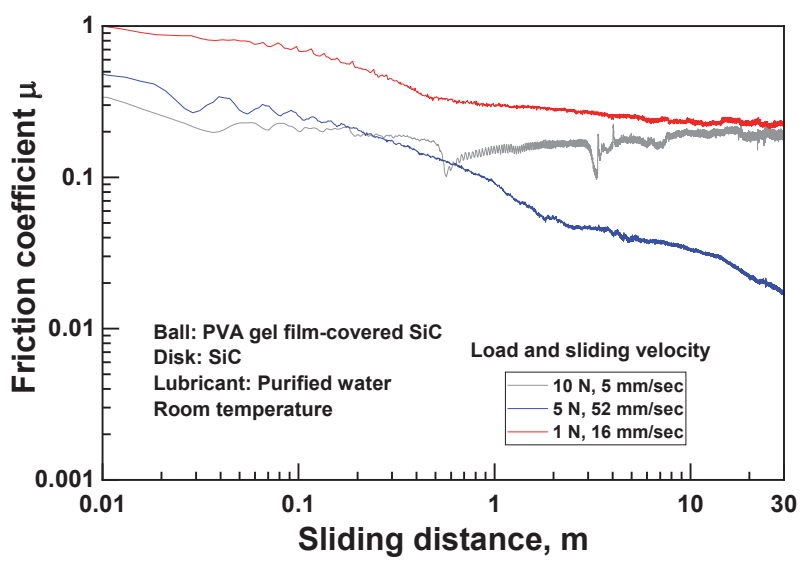

Fig. 4 Typical friction properties of PVA gel and SiC disk in water

3.2 Wear scar formed on gel film

A typical DN gel film surface after various experimental conditions with each friction mode is shown in Fig. 5.

Apparent linear wear scars along the sliding direction of the counter surface are seen on the DN gel film after mode I. In addition, slight linear wear scars are seen on the DN gel surface after modes II, III, and IV. Also residual concaves still can be seen on the surface after mode II, III. Specifically, after mode IV, apparent linear wear scars are hardly observed on the surface of the DN gel by used optical microscope in this study.

Drastic wear surface after mode I is understood by adhesion between $\mathrm{SiC}$ disk and DN gel surface in boundary lubrication regime due to high load and slow sliding speed region. On the other hand, in the case of mode IV, both surfaces could be separated completely from the beginning of friction test, thus apparent wear scar was not made on DN gel surface.

The PVA gel film surface after each mode is shown in Fig. 6. Relatively rougher surfaces are seen after all three modes compared to the examined DN gel surfaces shown in Fig. 5. In the case of modes I and II, severe wear scars were observed on both PVA gel surfaces. After mode III, linear wear scars were seen along the sliding direction of the counter surface as well as on the DN gel surface but that frequency is more often compared to that of DN gel. Fundamentally, tensile strengths of DN gel and PVA gel are reported as up to $10 \mathrm{MPa}$ and sub-MPa respectively [15], therefore it is understandable that PVA gel is worn severely compared to $\mathrm{DN}$ gel surface even if friction mode is similar.

\subsection{Distribution of friction mode}

The distributions of the friction mode, with the function of the sliding speed and load, are shown in Fig. 7 for both DN gel and PVA gel. When the DN gel film is examined, mode I appears at a relatively lower sliding speed and higher load. Modes IV occur at a relatively lower contact load compared to modes II and III. The friction mode transits from II to III and from III to IV as the sliding speed becomes faster.

When the PVA gel film is examined, mode I widely occurs at a higher sliding speed compared to that of the DN gel. The region where mode II occurs shifts to a higher sliding speed. Modes III and IV do not take place within the examined experimental conditions.

Friction coefficient at saturated state as a function of sliding speed divided by load is shown in Fig. 8. Plots with a 


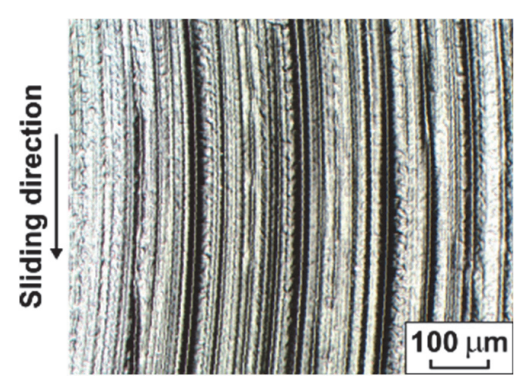

(a) mode I

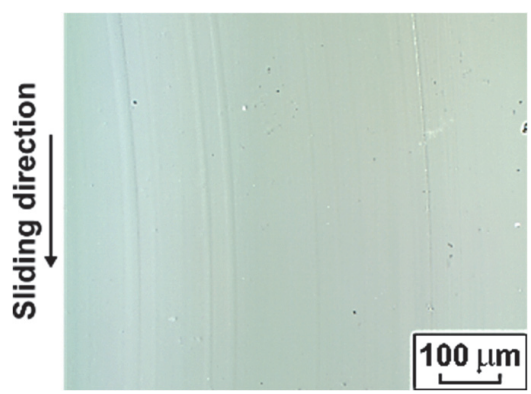

(c) mode III

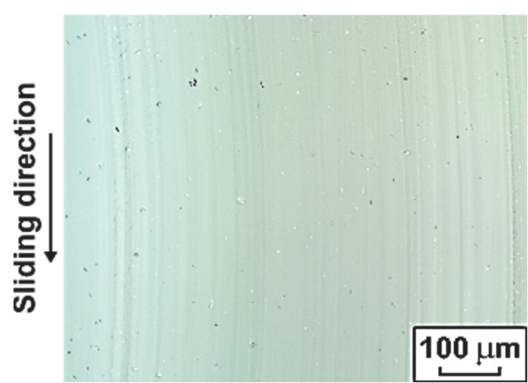

(b) mode II

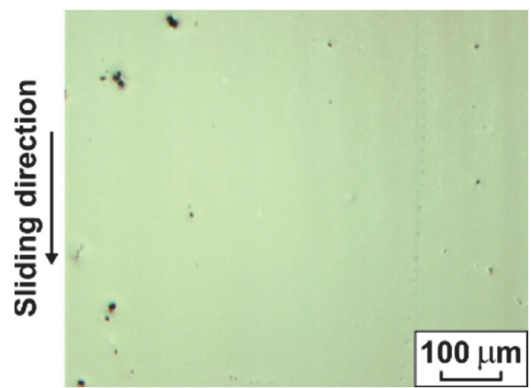

(d) mode IV

Fig. 5 Typical DN gel surface after each friction modes

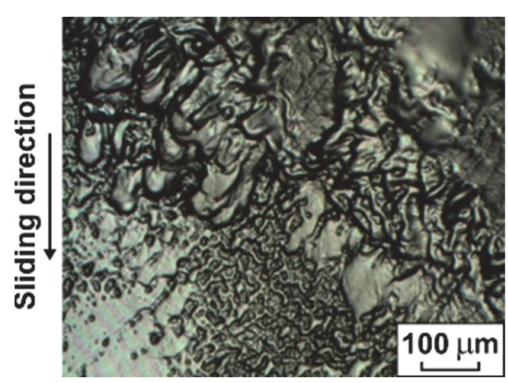

(a) mode I

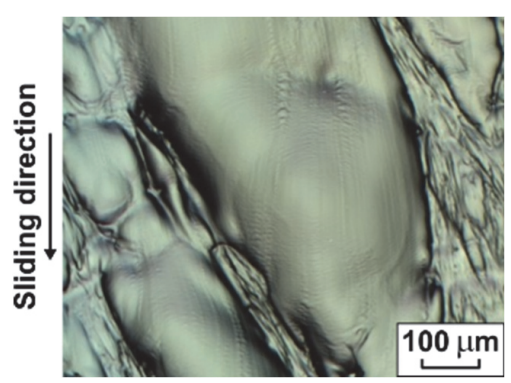

(b) mode II

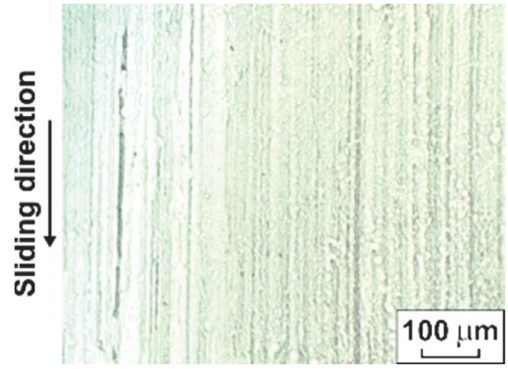

(c) mode III

Fig. 6 PVA gel surface which shows (a) mode I, (b) mode II and (c) mode III

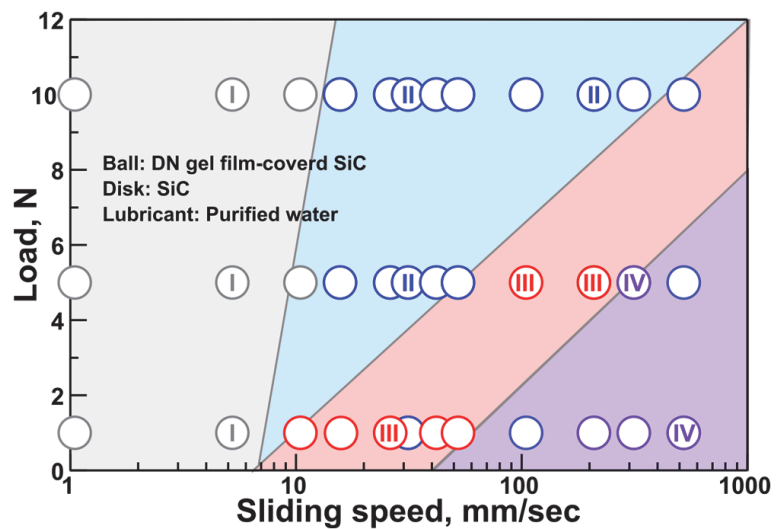

(a) DN gel

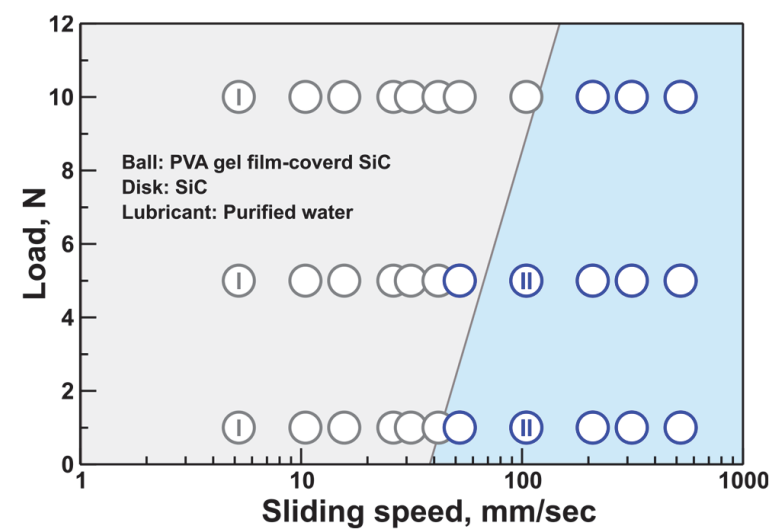

(b) PVA gel

Fig. 7 Distribution of the friction mode when (a) DN gel and (b) PVA gel are examined

down-pointing arrow indicate that friction coefficient is lower than detection limit of used test apparatus. As the value of the $\mathrm{x}$-axis increases, modes I, II, III and IV appear in order. It is understandable that high friction and severe wear are realized at boundary lubrication regime, while unmeasurable low friction and almost no wear are realized at hydrodynamic lubrication regime as understood by Stribeck curve.

Interesting point is that mode III, which shows an unique 


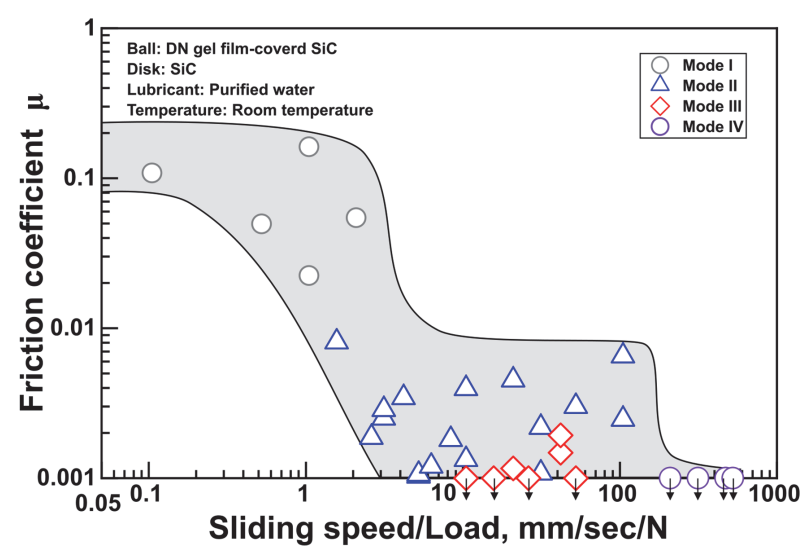

Fig. 8 Saturated friction coefficient as a function of sliding speed divided by load

decrease in the friction coefficient twice, is located between modes II and IV. Also, wear grade of mode III shows medium property between II and IV. These results indicate that the wear caused by certain friction severity is needed in order to generate running-in behavior shown in mode II. This leads to the consideration that the surface change of the DN gel due to the applied stress realizes mode III.

\section{Discussions}

4.1 Formation of damaged zones on the DN gel surface

Friction experiments were carried out in a fluorescent particle solution (PUS-102T, MITSUBISHI PENCIL CO., LTD.) and cross-sections of the sliced DN gel were observed by fluorescence microscopy (DINOAM4115TGFBW, ANMO Electronics Corp.). We found that diameter of fluorescent particle was $100 \mathrm{~nm}$ approximately by SEM observation. Fluorescence solution is able to penetrate into DN gel due to its higher osmotic pressure than that of purified water. The sliding speed of the counter $\mathrm{SiC}$ disk was $42 \mathrm{~mm} / \mathrm{sec}$ and sliding distance was set to be $30 \mathrm{~m} .1 \mathrm{~N}$ and $10 \mathrm{~N}$ forces were chosen as contact loads to simulate modes II and III especially. In addition, a static immersion test of the DN gel film was carried out in a fluorescent particle solution to highlight the effect of friction on the penetration of external water into the $\mathrm{DN}$ gel.

Distributions of penetrated fluorescent particles in the DN gel are shown in Fig. 9. The distribution of these particles is shown as a yellow region and its brightness is related to the volume of fluorescent ink. In the DN gel after static immersion tests, fluorescent particles are observed on the DN gel surface slightly and are hardly distributed as shown in Fig. 9 (a). On the other hand, when the DN gel experiences friction in a fluorescent particle solution, fluorescent particles are distributed in the DN gel deeper than $500 \mu \mathrm{m}$ from the top of the surface. Furthermore, an apparent brighter layer is formed beneath the DN gel surface with a thickness of $100 \mu \mathrm{m}$ approximately. Thus contribution of friction on penetration of external water in DN gel was clearly shown by these experimental results.

The DN gel is known to form a damaged zone with a thickness of several hundred nm beneath the top of the surface discontinuously due to the applied stress [14]. Also, the structure of the damaged zone is found to be soft and some networks are partially broken compared to their initial state [14]. It is estimated that the water between the DN gel surface and its sliding counterpart, marked by fluorescent particles, penetrated

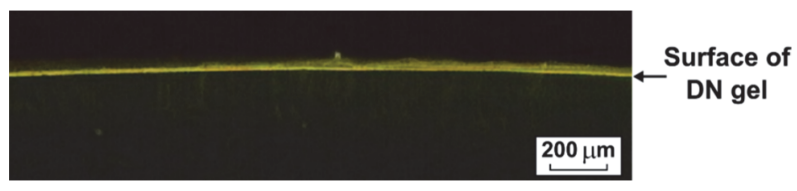

(a) static immersion test

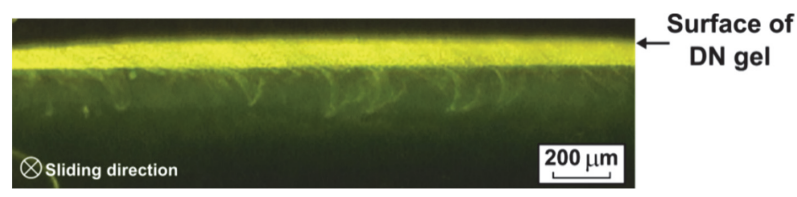

(b) mode II

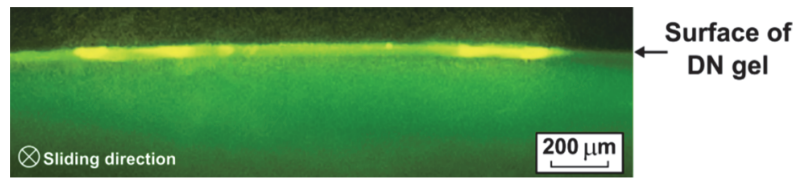

(c) mode III

Fig. 9 Distribution of fluorescence ink at cross-section of DN gel after (a) static immersion test and friction mode shows (b) mode II and (c) mode III

the damaged zone of the DN gel. As a result, the water content in the damaged zone is enriched and the water moves inside of the DN gel and goes even deeper, where the damaged zone is not formed. These results indicate that friction can change the structure of the DN gel surface and new surface properties can be given to this surface.

\subsection{Formation process of damaged zone}

By comparing chemically-made hydrogels which surface has lower and higher polymer volume fraction, it was clarified that lubricious water layer can be kept by lower fraction than higher fraction [12]. Polymer volume fraction density at selfformed damaged zone is estimated to be lower than bulk DN gel, therefore self-formed damaged zone is crucial to realize mode III and formation process will be analyzed.

In-situ friction apparatus was introduced to detect the behavior of water and DN gel surface through each friction mode. In addition, colored water and colored DN gel enable the observation of water behavior. The schematic of the insitu friction apparatus is shown in Fig. 10 (a). The friction experiment of the DN gel against a transparent glass plate in water, including red color ink, was performed. The DN gel was colored by green ink before the friction experiment to highlight the behavior of red water. The DN gel surface was observed by a high-speed camera (VW-9000, Keyence) and lens (VH-Z20T, Keyence) through a transparent glass plate. The mechanical components used to measure friction, such as a pivot and a load cell are shown in Fig. 2, and are also used in the in-situ test apparatus.

The initial image of the loaded DN gel through the transparent glass plate is also shown in Fig. 10 (b). White circular lines indicate the border of contact between the deformed DN gel and the glass plate. White linear scars are also formed on the opposite side of the glass plate and are not involved in the friction process against the DN gel. Also, the reflection of a light source is seen as a circumferential white region.

In-situ observation images of the DN gel when the friction behavior corresponds to modes I, II, and III are shown in Fig. 


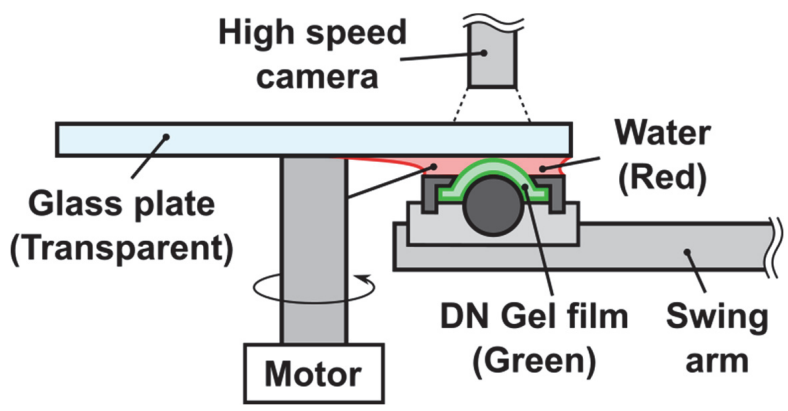

(a) Schematic

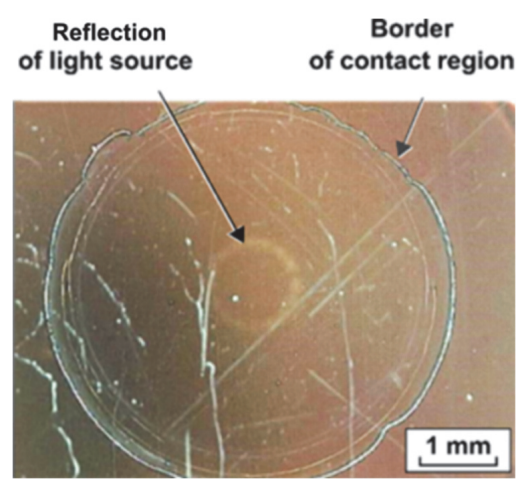

(b) initial image DN gel in contact

Fig. 10 (a) Schematic of in-situ friction apparatus and (b) initial image of DN gel in contact

11 respectively. In the case of mode $\mathrm{I}$, the DN gel around the contact region appears to be green, which originated from the colored DN gel. Thus the phenomenon in mode I was clarified as direct contact of DN gel and glass plate is dominant phenomena due to boundary lubrication slower sliding speed and higher contact pressure.

Since the red color of the water and the green color of the DN gel are complementary, mixed red and green solution will show black color. Therefore dark color shown in the case of mode III, shown Fig. 11 (c), clarifies external red water penetrated into green-colored DN gel surface. The phenomena that occurred in mode II will be discussed further with a detailed in-situ analysis of the DN gel surface during its generation process.

Typical fiction behaviors achieved by an in-situ tribometer are shown in Fig. 12. Similar friction behaviors are seen as compared to Fig. 3 and modes I, II, and III appeared similarly although the experiment details were different, such as the absolute value of friction coefficient or sliding distance. The following phases were defined based on the friction behavior of mode III and is shown in Fig. 12.

Phase A : Beginning of the friction experiment and the friction coefficient starts to decrease.

Phase B : Friction coefficient saturates after the first decrease.

Phase C : Friction coefficient shows stable value.

Phase D : Friction coefficient starts to show a second decrease.

Phase E: Friction coefficient saturates after the second decrease.

A series of DN gel surface images at phases A, B, C, D, and $\mathrm{E}$ are shown in Fig. 13. At phase $\mathrm{A}$, a roughening region is seen

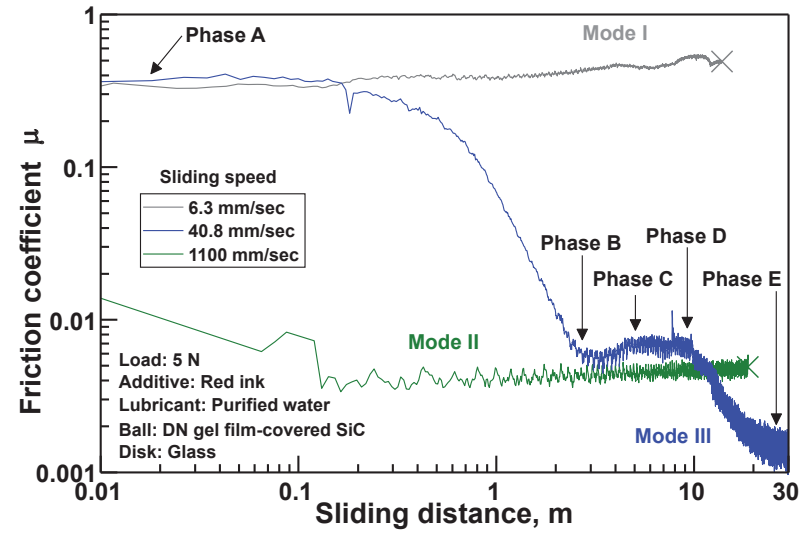

Fig. 12 Typical fiction behaviors achieved by in-situ tribometer

as a curved white belt on the top of the DN gel contact region. In addition, linear wear scars are formed below the white belt region along the sliding direction of sliding glass plate. A green region, where the red external water disappeared between the glass plate and the DN gel surface, is seen above the white belt region. As the phase shifts from A to B, the curved white belt region moves lower along the sliding direction of the counter surface. At phase $B$, the contact region is shrunk at the bottom of the initial contact region from phase A and more apparent linear wear scars are seen in the localized contact region. In addition, red water flows between the $\mathrm{DN}$ gel and the glass plate from above. From phase B to $\mathrm{C}$, the localized contact region still moves lower and shrinks. Moreover, the red water continues to flow between the DN gel and its sliding counterpart. At phase D,

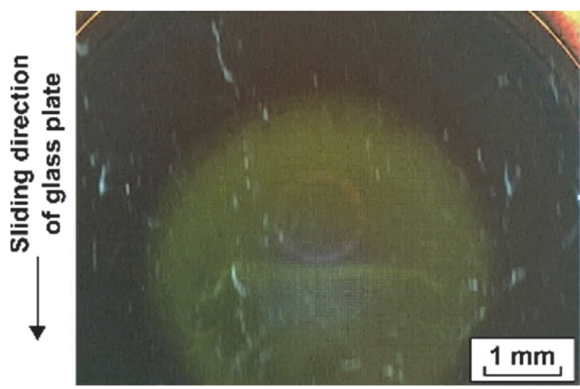

(a) mode I

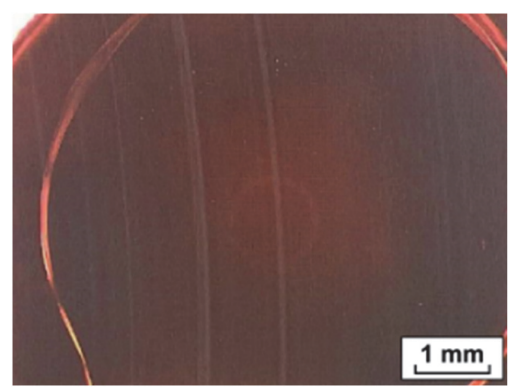

(b) mode II

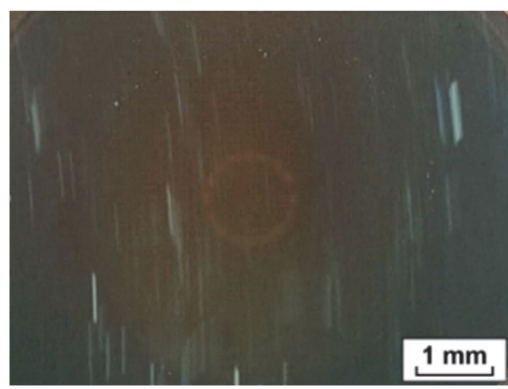

(c) mode III

Fig. 11 Typical images of a in-situ sliding interface at a saturated state of (a) mode I, (b) mode III, and (c) mode III 


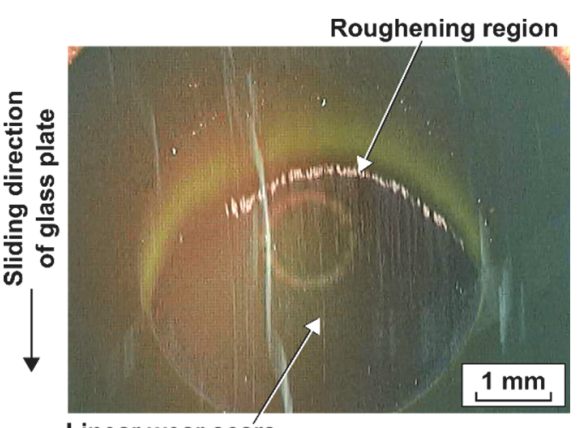

Linear wear scars

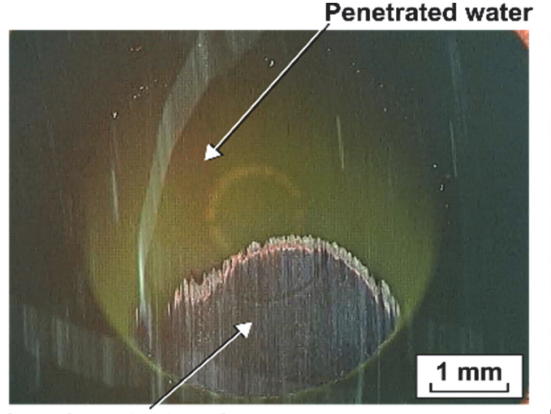

Local contact region

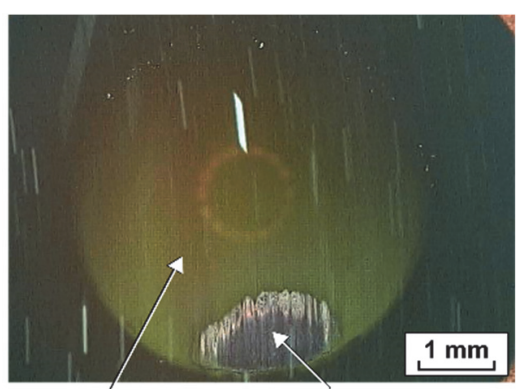

Penetrated water Shrieked contact region

(c) phase $\mathrm{C}$

(a) phase $\mathrm{A}$

(b) phase B

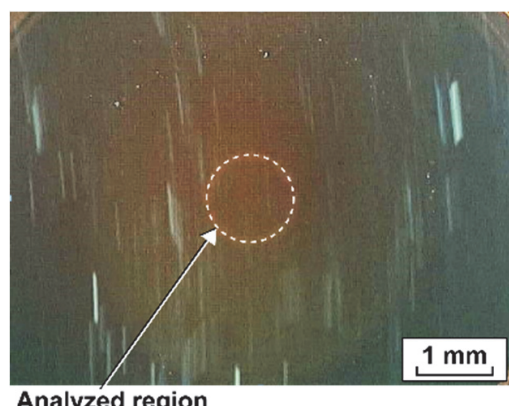

Analyzed region

(d) phase D

(e) phase E

Fig. 13 In-situ observation of DN gel at (a) phase A, (b) phase B, (c) phase C, (d) phase D, and (e) phase E in mode III

the localized contact region disappears and the DN gel starts to have uniform contact with its sliding counterpart. From phases $\mathrm{D}$ to $\mathrm{E}$, the whole region gets darker.

Based on the DN gel images and friction properties, the first decrease of the friction coefficient in mode III is estimated as follows: the local contact region between the DN gel and its sliding counterpart dominates the friction process due to adhesion and the friction coefficient decreases as contact area causes. Finally, applied tensile stress by sliding counterpart forms damaged zone automatically beneath the DN gel surface.

From phase D to phase E, where the friction coefficient shows a second decrease, the red intensity at the center of the contact region on the DN gel was analyzed every $5 \mathrm{~m}$ of sliding distance. The count transition of the red intensity is shown in Fig. 14. The red intensity has a broad distribution and shows a peak value at approximately 110 steps at phase D in mode III. Then the peak value of the red intensity count shifts lower as the sliding distance increases from phase D to E. Finally, a peak position is observed at 95 steps approximately, when the sliding distance is $30 \mathrm{~m}$ (at phase E). Since the mixture of the red and green ink shows a black color on the basis of the subtractive mixture, the darkening of the DN gel images from phase $\mathrm{D}$ to $\mathrm{E}$ indicate that water including red ink penetrates more and more into the DN gel surface.

The generation mechanism of mode III is estimated as follows:

The damaged zone is formed beneath the DN gel surface when this surface is roughening from phase A to phase D due to shear.

Furthermore, the water between the DN gel surface and its sliding counterpart penetrates into the damaged zone and the water concentration at this region becomes higher than initial state. As explained by previous research [12], water-rich region

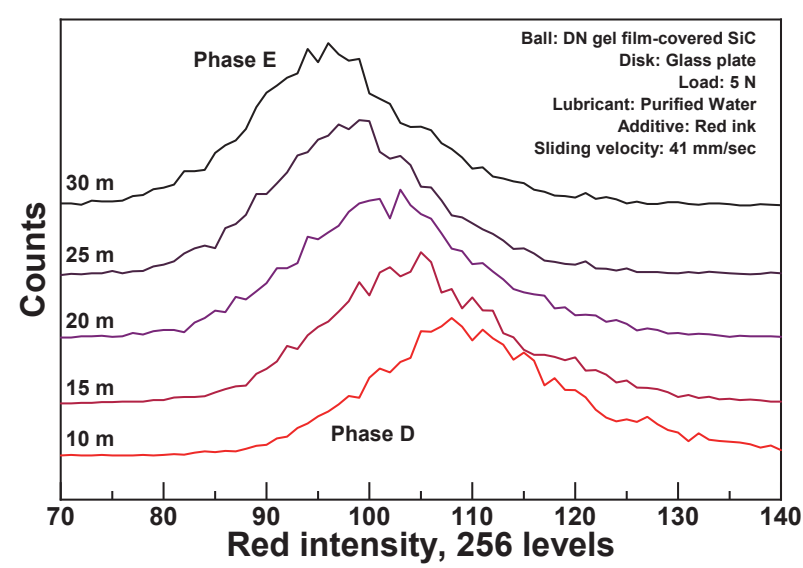

Fig. 14 Transition of red scale at the contact region of the DN gel

in hydrogel enables to keep water interface than water-depleted surface, thus damaged zone is believed to form water interface automatically through generation process of mode III. Driving force for water absorption into DN gel is estimated to be osmotic pressure caused by expanded space in DN gel formed by broken networks.

After phase D, the damaged zone creates a water layer, which suppresses the direct contact between the DN gel networks and sliding counterparts; thus a second decrease of the friction coefficient occurs as the water concentration of the damaged zone is enriched more and more. However, since the damaged zone is also formed on the DN gel surface the case in mode II as shown in Fig. 9 (b), the formation of the damaged zone is a necessary but is not sufficient to cause the double 
decrease of the friction coefficient. Since mode III occurs under relatively smaller load conditions compared to mode II, a larger contact load can disable the role of the damaged zone to attract a water layer since higher contact pressure can push out water from contact interface as well as from inside of DN gel. Also, higher mechanical toughness of the DN gel prevents the wear of damaged zones and the remaining damaged zones continue to form a thin water layer.

\section{Conclusions}

Friction tests using a DN gel film and a SiC disk or glass plates were conducted in water and the following conclusions were achieved.

(1) The friction coefficient in mode III shows 0.1 at the beginning and decreases around 0.01 , then decreases again to lower than 0.001 . Thus the potential of the DN gel to generate a low friction by running-in is clarified.

(2) Self-formed damaged zone keeps water layer and external water penetrates into damaged zone as sliding distance increases, thus lubricious water layer dominates friction between DN gel and sliding counterpart. As a result, friction coefficient shows $2^{\text {nd }}$ decrease in mode III. Thus potential of DN gel to realize low friction by running-in was clarified.

\section{Acknowledgments}

The authors express their gratitude to Prof. Hidemitsu Furukawa in Yamagata University for his helpful introduction of the DN gel synthesis. The authors would also like to thank Dr. Yu Kaihong at the Institute of Fluid Science, Tohoku University, for his dedicated work on the PVA gel synthesis.

\section{References}

[1] Blau, P. J., "On the Nature of Running-in," Tribology International, 38, 11-12, 2005, 1007-1012.

[2] Zambrano, O. A., Muñoz, E. C., Rodríguez, S. A. and Coronado, J. J., "Running-in Period for the Abrasive Wear of Austenitic Steels," Wear, 452-453, 2020.

[3] Wang, X., Kato, K. and Adachi, K., "Running-in Effect on the LoadCarrying Capacity of a Water-Lubricated SiC Thrust Bearing," Proceedings of the Institution of Mechanical Engineers, Part J: Journal of Engineering Tribology, 219, 2, 2005, 117-124.

[4] Chen, M., Kato, K. and Adachi, K., "Friction and Wear of Self-Mated $\mathrm{SiC}$ and $\mathrm{Si}_{3} \mathrm{~N}_{4}$ Sliding in Water," Wear, 250, 1-12, 2001, 246-255.

[5] Deng, M., Li, J., Zhang, C., Ren, J., Zhou, N. and Luo, J., "Investigation of Running-in Process in Water-Based Lubrication Aimed at Achieving Super-Low Friction," Tribology International,
$102,2016,257-264$.

[6] Kano, M., Yasuda, Y., Okamoto, Y., Mabuchi, Y., Hamada, T., Ueno, T., Ye, J., Konishi, S., Takeshima, S., Martin, J. M., De Barros Bouchet, M. I. and Le Mognee, T., "Ultralow Friction of DLC in Presence of Glycerol Mono-Oleate (GMO)," Tribology Letters, 18, 2, 2005, 245-251.

[7] Mikložič, K. T. and Spikes, H. A., "Application of Atomic Force Microscopy to the Study of Lubricant Additive Films," Journal of Tribology, 127, 2, 2005, 405-415.

[8] Akbarzadeh, A. and Khonsari, M. M., "Effect of Untampered Plasma Coating and Surface Texturing on Friction and Running-in Behavior of Piston Rings," Coatings, 8, 3, 2018, 110.

[9] Kopeček, J., "Swell Gels,” Nature, 417, 2002, 389-391.

[10] Zhang, A., Chen, M., Du, C., Guo, H., Bai, H. and Li, L., "Poly(Dimethylsiloxane) Oil Absorbent with a Three-Dimensionally Interconnected Porous Structure and Swellable Skeleton," ACS Applied Materials \& Interfaces, 5, 20, 2013, 10201-10206.

[11] Stammen, J. A., Williams, S., Ku, D. N. and Guldberg, R. E., "Mechanical Properties of a Novel PVA Hydrogel in Shear and Unconfined Compression," Biomaterials, 22, 8, 2001, 799-806.

[12] Gombert, Y., Simič, R., Roncoroni, F., Dübner, M., Geue, T. and Spencer, N. D., "Structuring Hydrogel Surfaces for Tribology," Advanced Materials Interfaces, 6, 22, 2019, 1901320.

[13] Simič, R., Yetkin, M., Zhang, K. and Spencer, N. D., "Importance of Hydration and Surface Structure for Friction of Acrylamide Hydrogels," Tribology Letters, 68, 2, 2020, 64.

[14] Shoaib, T. and Espinosa-Marzal, R. M., "Advances in Understanding Hydrogel Lubrication," Colloids and Interfaces, 4, 4, 2020, 54.

[15] Gong, J. P., “Why are Double Network Hydrogels so Tough?," Soft Matter, 6, 12, 2010, 2583-2590.

[16] Ren, H. Y., Mizukami, M., Tanabe, T., Furukawa, H. and Kurihara, K., "Friction of Polymer Hydrogels Studied by Resonance Shear Measurements," Soft Matter, 11, 31, 2015, 6192-6200.

[17] Gong, J. P., "Friction and Lubrication of Hydrogels-Its Richness and Complexity," Soft Matter, 2, 7, 2006, 544-552.

[18] Kosukegawa, H., Mamada, K., Kuroki, K., Liu, L., Inoue, K., Hayase, T. and Ohta, M., "Measurements of Dynamic Viscoelasticity of Poly (Vinyl Alcohol) Hydrogel for the Development of Blood Vessel Biomodeling," Journal of Fluid Science and Technology, 3, 4, 2008, 533-543.

[19] Tanaka, Y., Kawauchi, Y., Kurokawa, T., Furukawa, H., Okajima, T. and Gong, J. P., "Localized Yielding around Crack Tips of DoubleNetwork Gels," Macromolecular Rapid Communications, 29, 18, 2008, 1514-1520.

[20] Kosukegawa, H., Fridrici, V., Laurenceau, E., Kapsa, P. and Ohta, M., "Friction of 316L Stainless Steel on Soft-Tissue-Like Poly(Vinyl Alcohol) Hydrogel in Physiological Liquid," Tribology International, 82, Part B, 2015, 407-414.

This paper is licensed under the Creative Commons Attribution-NonCommercial-NoDerivatives 4.0 International (CC BYNC-ND 4.0) License. This allows users to copy and distribute the paper, only upon conditions that (i) users do not copy or distribute such paper for commercial purposes, (ii) users do not change, modify or edit such paper in any way, (iii) users give
ith a link to the formal publication through the relevant DOI (Digital Object Identifier)) and provide a link to this license, and (iv) appropriate credit (with a link to the formal publication through the relevant DOI (Digital Object Identifier)) and provide a link to this license, and (iv)
users acknowledge and agree that users and their use of such paper are not connected with, or sponsored, endorsed, or granted official status by the Licensor (i.e. Japanese Society of Tribologists). To view this license, go to https://creativecommons.org/licenses/by-nc-nd/4.0/. Be noted that the third-party materials in this article are not included in the Creative Commons license, if indicated on the material's credit line. The users must obtain the permission of the copyright holder and use the third-party materials in accordance with the rule specified by the copyright holder. 